\title{
Capacity Worth of Energy Storage System in Renewable Power Generation Plant
}

\author{
Jinbin $\mathrm{Li}^{1,2}$, Yao Yao ${ }^{1,2}$ \\ ${ }^{1}$ Hubei Electric Power Research Institute, Wuhan, China \\ ${ }^{2}$ Key Laboratory of High-Voltage Field-Test Technique of SGCC, Wuhan, China \\ Email: qq369178871@126.com
}

Received May 2013

\begin{abstract}
With the advance in renewable generation technologies, the cost of renewable energy becomes increasingly competitive when compared to fossil fuel-based generation resources. It is economically beneficial to integrate large amounts of renewable capacity in power systems. Unlike traditional generation facilities, however, using renewable resources for generation presents technical challenges in producing continuous power. In this report, an Energy Storage System (ESS) is integrated to smooth the variations in renewable power production and ensure the output power more controllable. Since it requires capital investment for the storage devices, it is important to obtain reasonable estimate of the storage capacities. This project is therefore formulated as an optimization problem in determining the two dominating factors of the capital cost for the ESS: the power capacity and the energy capacity. The objective is to make the renewable power more reliable and simultaneously maximize the economic benefits that can be obtained from the scheme. To make the results more convincing, analyses in this report start with wind generation, for wind has greater variability and unpredictability than other renewable sources. Selection of ESS type is narrowed down to battery energy storage system (BESS) in the scheme. However, the methods presented here are suitable for any type of energy storage methods and are also useful for intermittent renewable energy resources other than wind.
\end{abstract}

Keywords: Wind Power; Energy Storage System; Power Capacity; Energy Capacity

\section{Introduction}

Economic growth and prosperity since the industrial revolution have, in large part, been due to the utilization of fossil fuels. The consumption of fossil fuels has nearly doubled every 20 years since 1900 [1]. However, the long-term large combustion of fossil fuels has caused undesirable environment impacts. The burning process emits large amount of carbon dioxide and other gases which cause the "greenhouse effect" and pollution [2]. And, even if we set aside worries about the environment problems fossil fuels have brought to us, it must be acknowledged that continuous and economically-priced fossil fuels are fast coming to an end.

One obvious way to solve the worldwide energy crisis problem is to find new primary sources. Amongst the alternatives, wind energy is very promising. Many countries have set goal for high penetration levels of wind generations. The annual growth rate of global installed wind power capacity has exceeded $26 \%$ since 1990 s [3].

However, one down side of wind power is its variability and relative unpredictability. The output of wind power system depends highly on the local natural environment which is hardly controllable. Hence this form of electricity generation would introduce more uncertainty into the power grid. This presents a challenge when integrating large scale wind power into electrical power networks. To counter this, a usual scheme is to use energy storage devices as an energy buffer. It leads to a reduction in the amount of spinning and standby power reserve needed to mitigate system frequency and voltage excursions due to the variations in the wind power output [4].

\section{Power in Wind and Wind Turbine Model}

\subsection{Power in the Wind}

The power in the wind is closely related to the wind speed, the relationship between them is presented as follows [5]:

$$
P_{w}=0.5 \rho \mathrm{A} v^{3}
$$

where $P_{w}$ is the power in the wind (W); $\rho$ is the air density $\left(\mathrm{kg} / \mathrm{m}^{3}\right) ; A$ is the cross-sectional area through which the wind passes $\left(\mathrm{m}^{2}\right)$; and $v$ is wind speed normal to $A$ $(\mathrm{m} / \mathrm{s})$.

One expression that is often used to characterize the impact of height on wind speed is the following [5]: 


$$
v_{1} / v_{2}=\left(H_{1} / H_{2}\right)^{\alpha}
$$

The friction coefficient $\alpha$ is a function of the terrain over which the wind blows. Usually, for rough approximation in somewhat open terrain, the rule-of-thumb is to take a value of $1 / 7$ for $\alpha$ [5].

In research activities, the most commonly used function for characterizing the statistics of wind speed is the Rayleigh probability density function, given by [5]:

$$
f(v)=\frac{2 v}{c^{2}} \exp \left[-\left(\frac{v}{c}\right)^{2}\right]
$$

where $c$ is called the scale parameter.

\subsection{Idealized Wind Turbine Power Curve}

To figure out how much energy the wind turbine can obtain by harnessing the wind, the performance characteristics of wind turbine must be known. The most important technical information for a specific wind turbine is its power curve, which shows the relationship between turbine generator's electrical output and wind speed. An idealized power cure is shown in Figure 1.

The relationship between the output wind power and the wind speed can be described as follows [5],

$$
P=\left\{\begin{array}{lr}
0 & 0 \leq v \leq v_{C} \text { or } v \geq v_{F} \\
\frac{1}{2} \rho A v^{3} & C_{P} v_{C} \leq v \leq v_{R} \\
P_{R} & v_{R} \leq v \leq v_{F}
\end{array}\right.
$$

where $v_{c}$ is the cut-in wind speed; $v_{R}$ is the rated wind speed; $v_{F}$ is the furling wind speed; $P_{R}$ is the rated power of the wind turbine generator. $C_{p}$ is the rotor efficiency, which is the faction of the wind's power that is extracted by the turbine blades.

\section{Approach to Determine the Capacity of ESS}

Utilizing Equations (3) and (4), the probability distribution of the wind power outputted from the wind turbine generator is thus available. Its profile is illustrated in

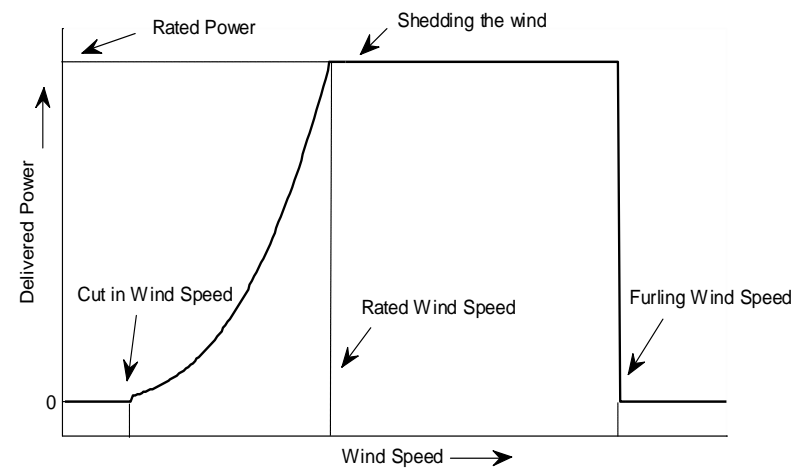

Figure 1. Idealized wind turbine power curve.
Figure 2. The probability increases sharply when the power reaches the rated value. This is due to the fact that the output power is limited to the rated power. Therefore, the probability increases sharply when one sums up all the probabilities that the wind speed ranges from $v_{R}$ to $v_{F}$.

The average of the generated power can be calculated as

$$
\bar{P}=\int_{0}^{P_{R}} \rho\left(P_{w}\right) d\left(P_{w}\right)
$$

In this project, it is proposed that the average power $\bar{P}$ will be set as the constant delivered power dispatched from the wind turbine station over the studied period. It will cause no net change in the stored energy in the ESS. The stored energy in the ESS will return to the same energy level at the end of the period. Generally, the average power in a short-term, say $24 \mathrm{hrs}$ ahead, can be estimated according to the local historical wind speed data or forecasting techniques. Thus, the intention of the constant delivered power is to ensure no net change in the stored energy in the ESS at the end of the subsequent 24 hrs.

The power capacity of the BESS can always be found as long as the probability distribution of the wind power is known and the average power is calculated. The power capacity can be equal to the larger of either the maximum charge power or the maximum discharge power, as shown on Figure 2.

\subsection{Method to Determine the Reduced Power Capacity of ESS}

Since the power capacity of the BESS is determined by the larger of the maximum charge and discharge power, therefore in certain situation, the power capacity may be equal to the maximum discharge power. Compared to the much higher probability at the rated power level, the probabilities of the low generated wind power level are much lower. While their contributions to the total generated energy are small, they have brought a notable increase in the power capacity of the BESS.

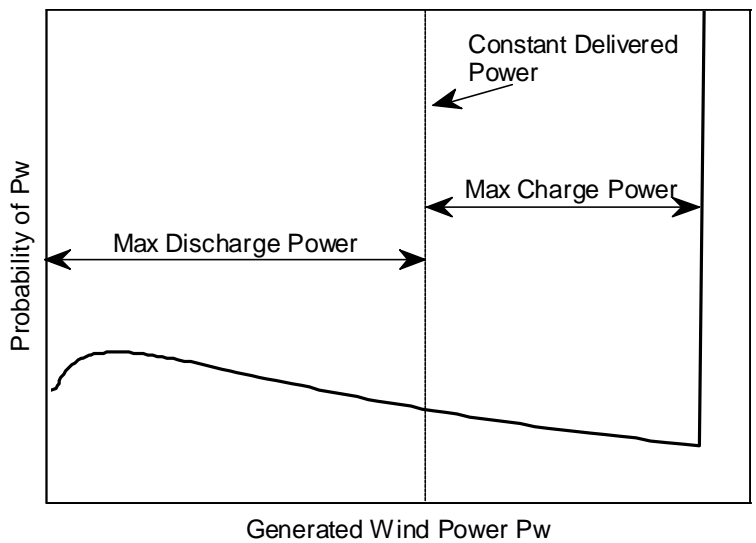

Figure 2. Probability distribution of generated wind power. 
It is now proposed that contributions from the low generated wind power components are delivered to a capacitor. This is because compared to the rated power, the energy contained in these components can be expected to be small. Furthermore, these components will cause frequent charge-discharge, it will not be desirable for the battery to deal with these charges $[6,7]$. Therefore, the author considers a capacitor a more suitable storage medium for these low power components. For those components of higher generated wind power levels, they are still to be dealt with by the BESS. The power network model is as shown in Figure 3.

By doing so, the lower limit of the BESS's discharging power would increase, and the average generated power from the BESS will also decrease most possibly slightly. Consequently the discharging capacity will decrease and the specified power capacity of the BESS may be reduced simultaneously. This decrease in the BESS power capacity will reduce the capital investment for the BESS, and thus may lead to an overall reduction in the cost for this wind power plant. This intention is illustrated in Figure 4, the darkened area refers to those low generated power instances which have been delivered to the capacitor storage.

The benefit of this method is a decrease in the amortized cost for the BESS's power capacity. Also, it can be expected that the energy capacity, which is another dominating factor of the capital cost for the BESS, will vary. To maximize the overall economic benefit that can be

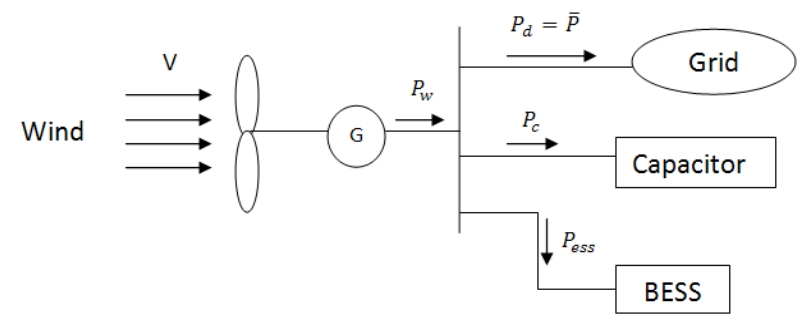

Figure 3. Power network model (BESS + Capacitor).

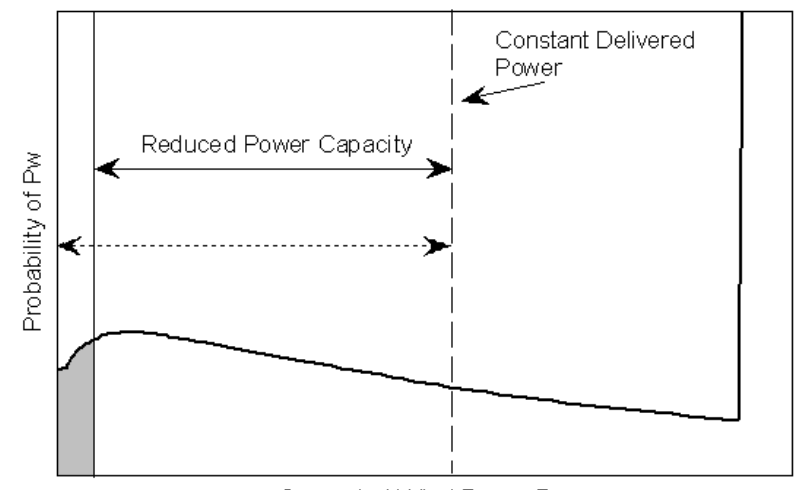

Generated Wind Power PW

Figure 4. Reduce the power capacity by delivering the low power components to the capacitor. obtained from this scheme, both the changes need to be taken into account to examine the economic effect and determine the optimal level of the low power components that should be assigned to the capacitor.

An analysis based on an actual site measurement rather than relying on Rayleigh assumptions is provided to examine the economic effects of this scheme.

\subsection{Analysis Based on Actual Site Measurement}

Data used in the following case study was recorded in Le Mars (Latitude 42.78, Longitude -96.2) at a reference height of $10 \mathrm{~m}$ on Nov 1st 2000 [8-10]. Figure 5 shows the probability distribution of the wind speed during the studied period.

To analyze the output power which can be harnessed from the wind resource, a typical idealized wind power-wind speed curve is assumed for the wind turbine. The parameters are set as $v_{c}=3 \mathrm{~m} / \mathrm{s}, v_{R}=12 \mathrm{~m} / \mathrm{s}, v_{F}=25 \mathrm{~m} / \mathrm{s}$, $P_{R}=2000 \mathrm{~kW}$, and the blade's radius $38 \mathrm{~m}$. The wind turbine is mounted with its hub at $50 \mathrm{~m}$ above the ground surface. Utilizing Equations (2) and (4), the probability distribution of the output power from the wind turbine is thus calculated, as shown in Figure 6.

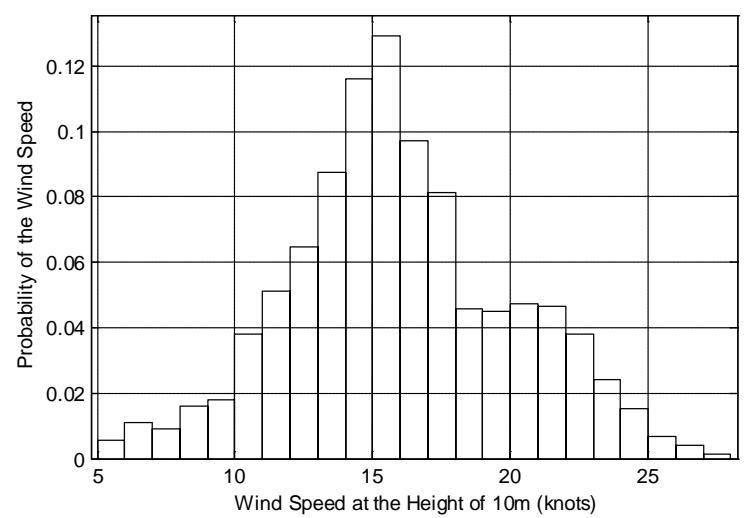

Figure 5. Probability distribution of the wind speed in Le Mars.

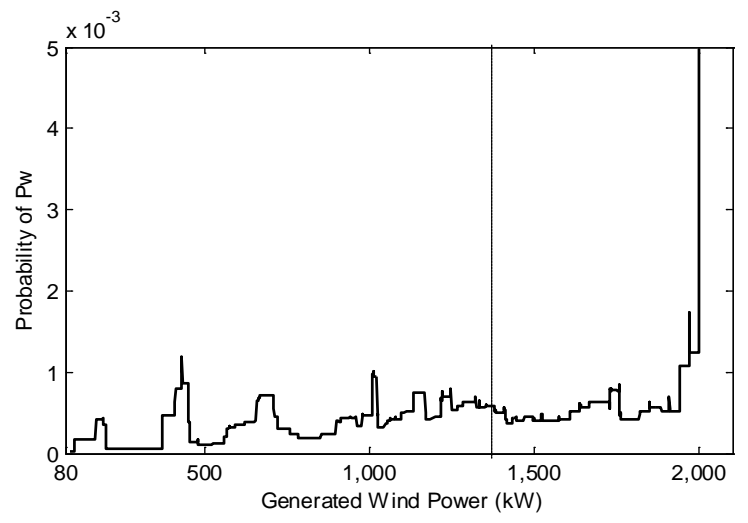

Figure 6. Probability distribution of the generated power in Le Mars. 
The average generated power is evaluated to be 1370.5 $\mathrm{kW}$. Thus, the BESS power capacity is $1370.5-80=$ $1290.5 \mathrm{~kW}$ (the probability of the generated power ranging from 0 to $80 \mathrm{~kW}$ is zero), according to the maximum discharge power.

Next, suppose the intention is to progressively deliver those contributions when the generated power level is low to the capacitor. The reason for doing so is to observe how by delivering the low power components to the capacitor will affect the power capacity of the storage element. The power level below which the contributions are delivered to the capacitor is denoted as $P_{c}$. Figure 7 shows how the power capacity of the BESS $C_{p}$ varies with $P_{c}$.

When $P_{c}$ increases successively, owing to the slight reduction in the average of the generated power, on one hand, the discharging capacity of the BESS decreases, on the other hand, the charging capacity increases simultaneously. It is reasonable to expect that at a certain $P_{c}$ value, the maximum charging capacity will be equal to the maximum discharging capacity, and beyond this value, the maximum charging capacity will exceed the maximum discharging capacity and the latter starts to dictate the BESS power capacity. This break point is shown in Figure 7.

After the low generated power components are assigned to the capacitor, the change in the energy capacity should be taken into account. Figure 8 shows how the energy capacity $C_{e}$ varies with $P_{c}$. The depth of the discharge is assumed to be $20 \%$ [11].

Assuming the wind power farm has $\mathrm{N}$ identical wind turbines, the life-time of the battery is $T$ (year). $\alpha$ $(\$ / \mathrm{kW})$ and $\beta(\$ / \mathrm{kWh})$ represent the coefficients of the amortized capital cost for the power capacity and energy capacity of the BESS, respectively. Coefficient $\gamma$ ( $\$ / \mathrm{kWh} /$ year) is used to represent the maintenance cost for the BESS each kWh of the energy capacity each year. Since the cost for the capacitor is really negligible when compared to the cost for the battery, the capital investment

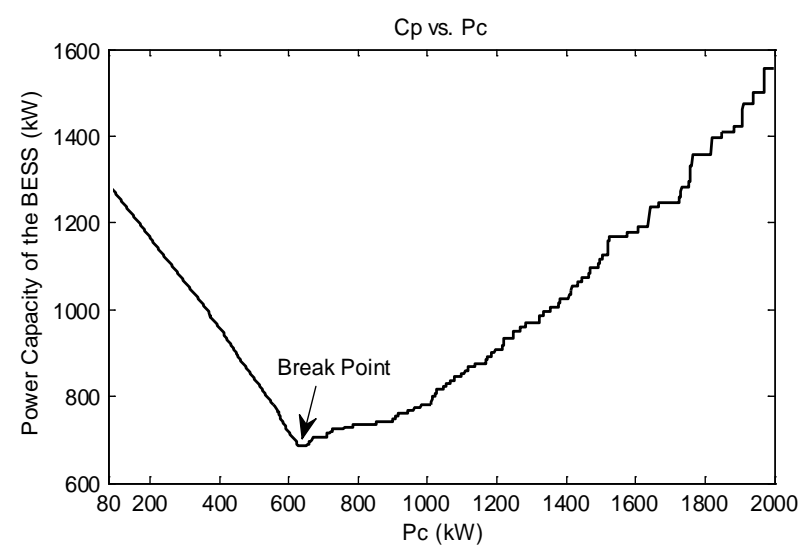

Figure 7. Power capacity $C_{p}$ of the BESS vs. $P_{c}$. for the capacitor is not taken into account. Then the total cost for the storage system can be described as:

$$
\begin{aligned}
& F(i)=N \cdot\left[\alpha \cdot C_{p}\left(P_{c}(i)\right)\right. \\
& \left.+\beta \cdot C_{e}\left(P_{c}(i)\right)+\gamma \cdot T \cdot C_{e}\left(P_{c}(i)\right)\right]
\end{aligned}
$$

For a predetermined battery type, $\alpha, \beta$, and $\gamma$ will be known. Thus, the total cost for the BESS of this method $F(i)$ can be evaluated, and the optimal power level $P_{c}$ will be found.

In this case study, the coefficients in (6) are selected in the following way. Suppose the wind farm has $\mathrm{N}=10$ identical wind turbines. The amortized power capacity cost of the BESS is such that $\alpha=203 \$ / \mathrm{kW}$. The amortized energy capacity cost of BESS $\beta=116 \$ / \mathrm{kWh}$. The battery life is 2 years, and the maintenance cost for the BESS per year is $\gamma=29 \$ / \mathrm{kWh}$ [12]. Figure 9 shows a plot of $F(i)$ based on these data.

According to the above figure, the capital cost for the BESS has a gentle decrease at the beginning. In this case, the best solution is to set $P_{c}=373 \mathrm{~kW}$, and then the capital cost for the BESS is about $\$ 1.2513 \times 10^{7}$. Compared to the capital cost of $\$ 1.2981 \times 10^{7}$ for the BESS before assigning those low generated power components to the capacitor, the method has resulted in a benefit of about $4 \%$.

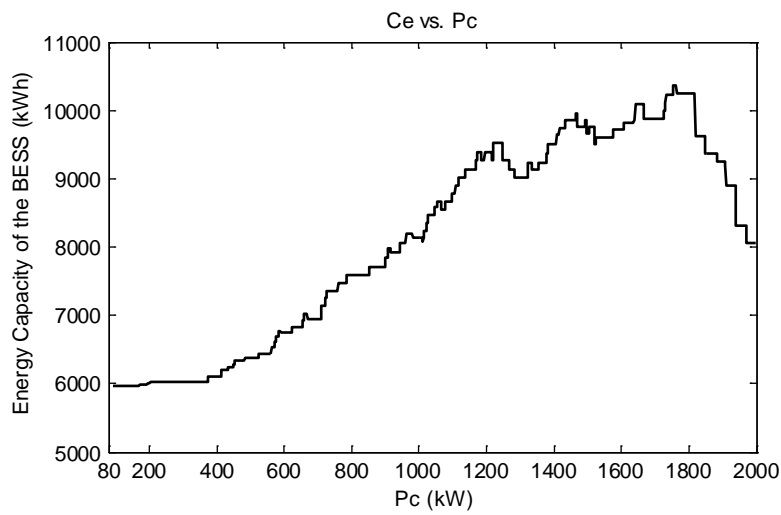

Figure 8. Energy capacity $C_{e}$ of the BESS vs. $P_{c}$.

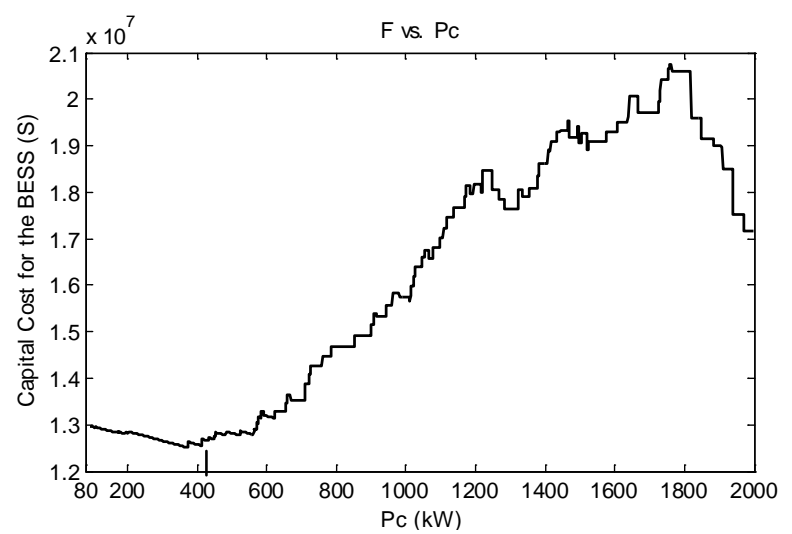

Figure 9. Capital cost for the BESS F vs. $P_{c}$. 
Using a similar process, other wind power plants could be analyzed. The optimal $P_{c}$ level can be found to minimize the capital cost for the BESS.

\section{Conclusion}

The methodology is proposed to assign those low generated power contributions to a capacitor. For those components of higher generated wind power levels, they are to be dealt with by the BESS to provide a constant delivered power for the grid. By doing so, the power capacity of the BESS would drop, and thus the capital cost for the BESS might probably decrease. The result from the overall design demonstrated that utilizing this method could create a considerable economic benefit.

\section{Acknowledgements}

The author wish to express his deepest gratitude and appreciation to his supervisor, Prof. Choi San Shing, for all his invaluable suggestion and patient guidance throughout the research period.

\section{REFERENCES}

[1] H. Lund, "Renewable Energy Systems," Elsevier Inc., San Diego, California, 2010.

[2] S. C. W. Krauter, "Solar Electric Power GenerationPhotovolataic Energy Systems,” Springer Inc., Rio de Janeiro, Brazil, Oct 2005.
[3] M. S. Lu, C. L. Chang, W. J. Lee and L. Wang, "Combining the Wind Power Generation System with Energy Storage Equipments,” IEEE, 2008.

[4] PEP (Professional Engineering Publishing), "Renewable Energy Storage-Its Role in Renewables and Future Electricity Markets,” John Wiley \& Sons Inc., 11 Jun 2008.

[5] G. M. Masters, "Renewable and Efficient Electric Power Systems,” John Wiley \& Sons Inc., Hoboken, New Jersey, 2004. http://dx.doi.org/10.1002/0471668826

[6] T. R. Crompton, "Battery of Reference Book," Butterworth International Edition, 1990, ISBN: 0-408-00791-5.

[7] D. L. Yao, "Battery Energy Storage System for a Wind Power Generation Scheme,” Master Dissertation, Nanyang Technological University, Singapore, 2008.

[8] "Wind Data Source: The lowa Department of Transportation (DOT)," URL. http://mesonet.agron.iastate.edu/request/awos/1min.php

[9] “National Renewable Energy Laboratory (NREL),” URL. http://www.nrel.gov/

[10] National Renewable Energy Laboratory, "National Solar Radiation Database 1991-2005 Update: User’s Manual,” Technical Report, NREL/TP-581-41364, April 2007.

[11] UNiROSS Industrial, “The Rechargeable Specialists,” URL.

http://www.uniross.com/UK/industrial/Cycle\%20life/010 102

[12] S. S. Choi, X. Y. Wang and D. M. Vilathgamuwa, "On the Design of Power Buffer and its Applications,” IEEE, DRPT2008, 6-9 April 2008, Nanjing, China. 